\title{
Gastric carcinoid tumors with aggressive lymphovascular invasion and lymph node metastasis
}

\author{
Kazuhiro Suganuma ${ }^{1}$, Yoshinide Otani ${ }^{1}$, Toshiharu Furukawa ${ }^{1}$, Yoshiro Saikawa ${ }^{1}$, Masashi Yoshida ${ }^{1}$, \\ Tetsuro Kubota ${ }^{1}$, Koichiro Kumai ${ }^{2}$, Kaori Kameyama ${ }^{3}$, Makio Mukai $^{3}$, and Masaki Kitajima ${ }^{1}$ \\ ${ }^{1}$ Department of Surgery, Keio University School of Medicine, 35 Shinanomachi, Shinjuku-ku, Tokyo 160-8582, Japan \\ ${ }^{2}$ Center for Diagnostic and Therapeutic Endoscopy, Keio University School of Medicine, Tokyo, Japan \\ ${ }^{3}$ Division of Diagnostic Pathology, Keio University School of Medicine, Tokyo, Japan
}

\begin{abstract}
We report a patient with multiple gastric carcinoid tumors without hypergastrinemia. An abdominal computed tomography (CT) scan was performed in a 66-year-old Japanese man who had abdominal discomfort. An abnormal, round, $2.5 \mathrm{~cm}$ mass close to the lesser curvature of the stomach was detected. Multiple small gastric carcinoid tumors were also detected by endoscopy. A total gastrectomy with lymph node dissection was performed after it was determined that the round mass was a lymph node metastasis of carcinoid tumor. Further pathological investigation of the surgical specimen revealed multiple gastric carcinoid tumors with severe lymphovascular invasion. The carcinoid tumors in the present patient were not related to hypergastrinemia. These lesions could not be grouped as any of the three types of gastric carcinoid tumors in the recent classification. Furthermore, as a simple distal gastrectomy is the standard treatment for multiple carcinoid tumors of the stomach, we recommend that a precise histopathological evaluation should be performed before an appropriate curative surgical treatment is selected.
\end{abstract}

Key words Carcinoid tumor - Lymphovascular invasion . Hypergastrinemia

\section{Introduction}

Gastric endocrine tumors are thought to arise from enterochromaffin-like (EC) cells in the gastric wall [1]. Ordinarily, these tumors proliferate slowly, and liver metastasis or lymph node metastasis only occurs in some cases. Gastric carcinoid tumors, a subclass of gastric endocrine tumors can be classified into three types [2-7,8]. Type I mainly consists of multiple tumors that have a close relationship to type A chronic atrophic

Offprint requests to: K. Suganuma

Received: April 16, 2003 / Accepted: July 14, 2003 gastritis and hypergastrinemia. Type II carcinoid tumors are found in patients with multiple endocrine neoplasia type 1 (MEN-1) and those with Zollinger-Ellison syndrome (ZES). This group of carcinoid tumors is also associated with hypergastrinemia, and multiple tumors are usually present. Type III carcinoid tumors are not associated with hypergastrinemia and usually appear as single large neoplasms with a high malignant potential. These tumors are usually comparatively large $(>1 \mathrm{~cm})$ and solitary. The incidence of these three types of carcinoid is about $80 \%, 6 \%$, and $14 \%$, respectively, for types I, II, and III. Furthermore, the incidence of multiple type 3 carcinoids is only $1 \%$ [9].

In the present study, we describe a patient with multiple carcinoid tumors but without hypergastrinemia, pernicious anemia, MEN-1, or ZES. Instead, lymphovascular invasion and lymph node metastasis were present; thus, the characteristics of the present case differ completely from those of the established groupings.

\section{Case report}

A 66-year-old man was admitted to our hospital by referral. He had visited his family doctor because of common-cold like symptoms and upper abdominal pain. Upper abdominal computed tomography (CT) was performed, and an abnormal mass was found in the lesser omentum as an extramural mass of the stomach.

A barium meal test revealed several elevated lesions that resembled submucosal tumors: one in the posterior wall of the upper body and two in the lower body (Fig. 1B). Upper gastrointestinal endoscopy revealed these elevated intragastric lesions to be submucosal tumors (Fig. 1A); a biopsy specimen was obtained, and the lesions were diagnosed as typical carcinoid tumors. The tumors were immunoreactive for chromogranin A and synaptophysin. Argentaffin granules were also observed in the cytoplasm of the tumor cells. Cellular atypia was 

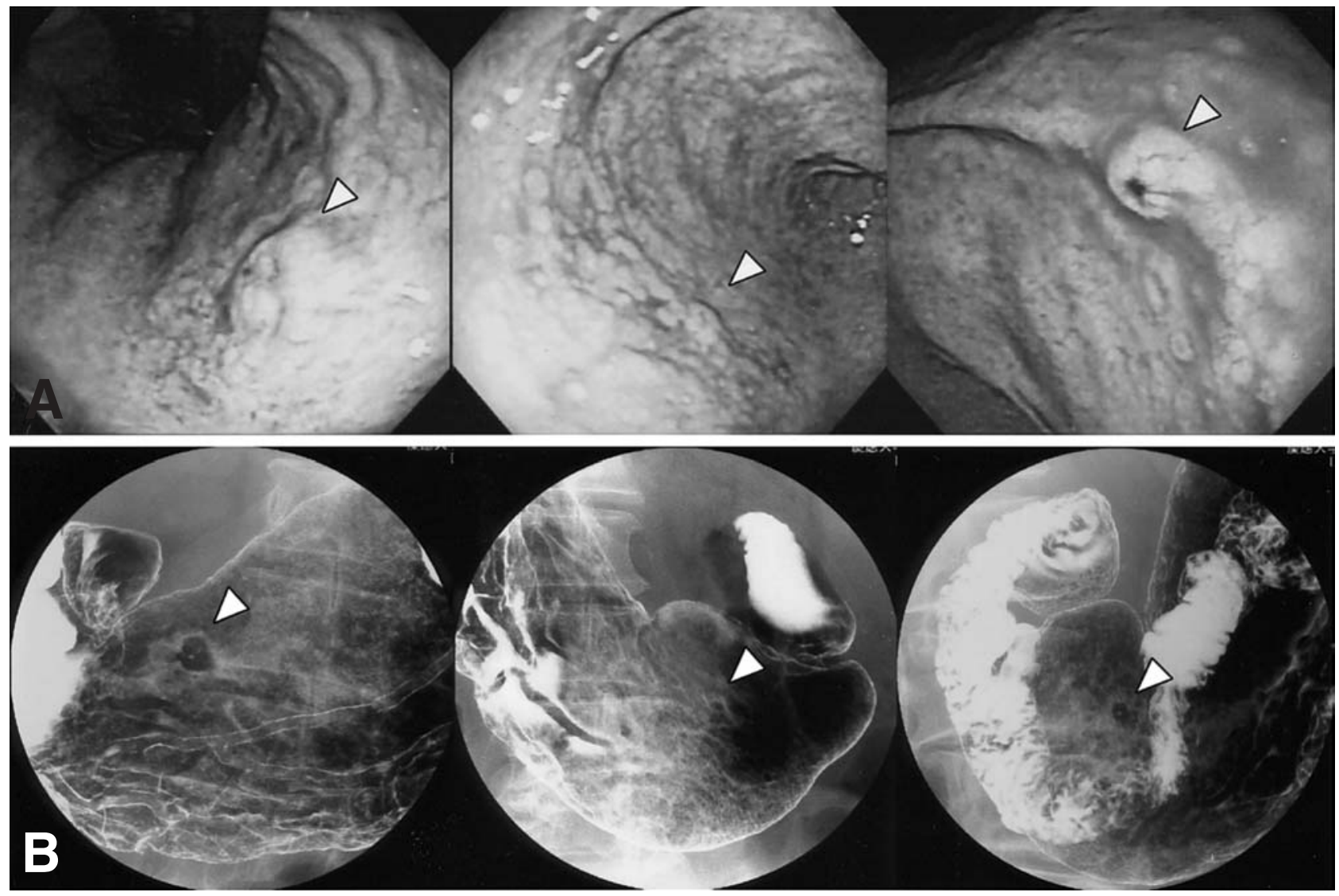

(1)

(2)

(3)

Fig. 1. A Endoscopic and $\mathbf{B}$ barium meal findings of the tumors. Three submucosal tumors are visible, in the lesser curvature (1), and in the anterior (2) and posterior walls (3) of the body of the stomach (arrowheads)

not prominent and mitoses were not observed. A barium enema showed multiple diverticuli, with a polyp size of $0.5 \mathrm{~cm}$ in diameter in the descending colon to a rectal polyp size of $0.3 \mathrm{~cm}$ in diameter. Digital examination and anal endoscopy failed to reveal any rectal polyps. Re-examination of the upper abdominal CT scan showed an enhanced gastric extramural mass, $2.5 \mathrm{~cm}$ in diameter; this was suspected of being a stromal tumor. No liver metastasis or paraaortic lymph node swellings were observed (Fig. 2). Abdominal ultrasonography (US) revealed only a mass $(3.0 \mathrm{~cm}$ in diameter) near the lesser curvature of the gastric body. No histological examination, such as an endoscopic ultrasonography (EUS)-guided needle biopsy, was performed for the extragastric mass. The patient was interviewed with regard to the presence of carcinoid symptoms, such as anemia, facial rash, and asthma-like attacks; none of these symptoms was present.

Among the preoperative laboratory examinations, the serum gastrin level was $83 \mathrm{pg} / \mathrm{ml}$ (normal range, 37
Table 1. Laboratory data

\begin{tabular}{|c|c|c|c|}
\hline WBC & $7300 / \mu l$ & CEA & $1.3 \mathrm{ng} / \mathrm{ml}$ \\
\hline $\mathrm{Hb}$ & $12.9 \mathrm{~g} / \mathrm{dl}$ & CA19-9 & $2 \mathrm{ng} / \mathrm{ml}$ \\
\hline Plt & $355000 / \mu \mathrm{l}$ & & \\
\hline Glu & $113 \mathrm{mg} / \mathrm{dl}$ & Gastrin & $83 \mathrm{pg} / \mathrm{ml}$ \\
\hline AST & $25 \mathrm{IU} / 1$ & $\begin{array}{l}\text { Anti-pariental cell } \\
\text { antibody }\end{array}$ & 20 Times \\
\hline ALT & 47 IU/1 & & \\
\hline $\mathrm{Na}$ & $140.6 \mathrm{mEq} / 1$ & Growth hormone & $7.7 \mathrm{ng} / \mathrm{ml}$ \\
\hline K & $4.2 \mathrm{mEq} / 1$ & Parathyroid hormone & $0.4 \mathrm{pg} / \mathrm{ml}$ \\
\hline $\mathrm{Ca}$ & $8.9 \mathrm{mEq} / \mathrm{l}$ & Catecholamine & WNL \\
\hline $\mathrm{TP}$ & $6.7 \mathrm{~g} / \mathrm{dl}$ & & \\
\hline Alb & $4.0 \mathrm{~g} / \mathrm{dl}$ & Urinalysis & \\
\hline UN & $19.9 \mathrm{mg} / \mathrm{dl}$ & $\mathrm{PH}$ & 6.0 \\
\hline $\mathrm{Cr}$ & $1.9 \mathrm{mg} / \mathrm{dl}$ & 5-HIAA & $1.0 \mathrm{mg} / \mathrm{day}$ \\
\hline
\end{tabular}

WNL, within normal limits

to $172 \mathrm{pg} / \mathrm{ml}$ ) and the serum catecholamine levels were also within the normal limits. The urinary 5hydroxyindole acetic acid (HIAA) level was $1.0 \mathrm{mg} /$ day (normal level, under $25 \mathrm{mg} /$ day). Pituitary and parathy- 

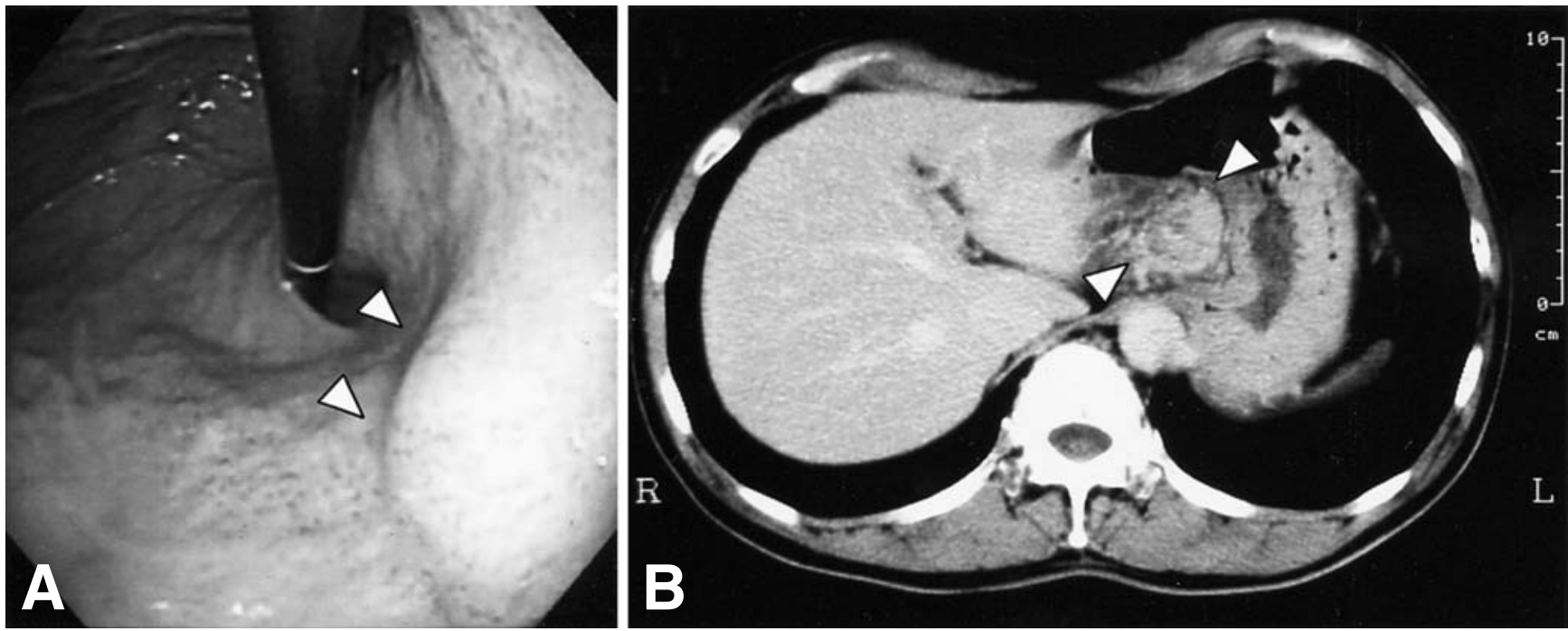

Fig. 2A,B. Findings of A endoscopy and $\mathbf{B}$ computed tomography (CT) scan of upper abdomen. A A submucosal tumor (SMT)like elevated lesion is observed in the lesser curvature of the body of the stomach (arrowheads). B CT scan revealed a gastric extramural mass, $2.5 \mathrm{~cm}$ in diameter (arrowheads), which was suspected of being a stromal tumor. No liver metastasis or paraaortic lymph node metastases are evident

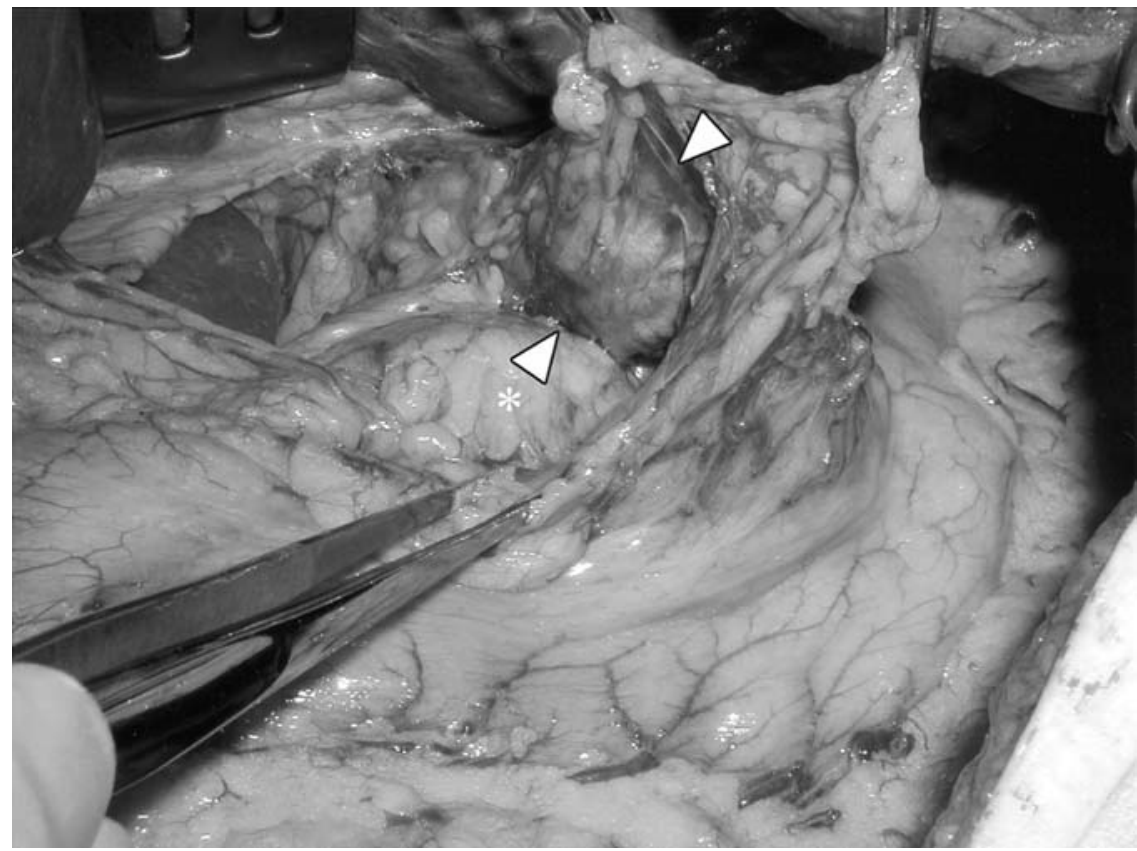

Fig. 3. Intraoperative pathological diagnosis revealed that the mass was a metastatic carcinoid tumor. Tumor cells have invaded the left gastric vein. Asterisk, extramural mass; arrowheads, lymph node roid hormone levels were also in the normal range. Anti-parietal cell antibody was positive at 1:20 (normal level, under $10 \times)$. Other laboratory data values were all within the normal limits (Table 1).

An operation was performed with the patient under general anesthesia. The operative findings and intraoperative pathological diagnosis revealed that the mass previously described as an extramural mass was, in fact, a lymph node metastasis of the carcinoid tumor. Only this lymph node was swollen, and the tumor cells had also invaded the left gastric vein (Fig. 3). Based on these findings, a total gastrectomy with $\mathrm{D} 1+\beta$ lymph node dissection was subsequently performed.

Macroscopically, the following elevated lesions were found: a 13-mm-diameter lesion in the lesser curvature 


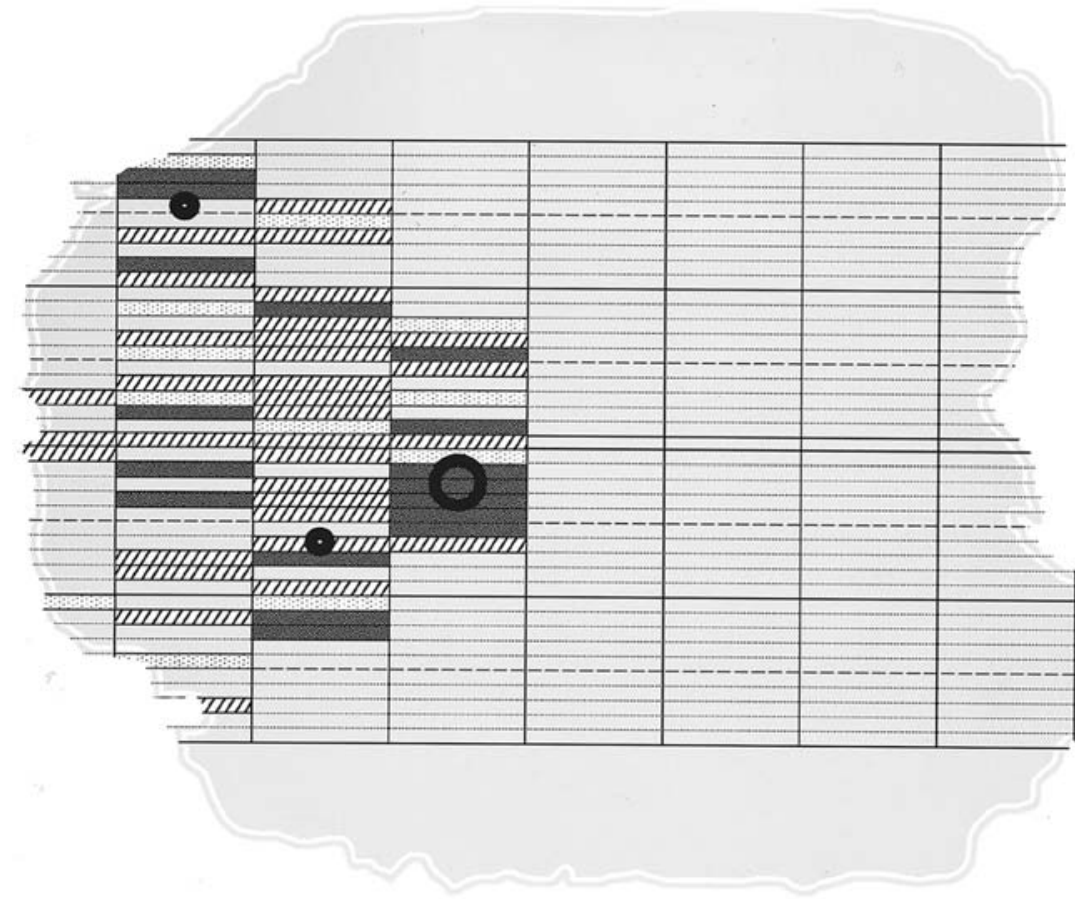

Fig. 4. Diagram of macroscopic findings. , slight vascular invasion; $\square$, moderate vascular invasion; $[$, severe vascular invasion; $\mathbf{O}$, carcinoid $(13 \mathrm{~mm})$; $\bullet$, carcinoid $(5 \mathrm{~mm})$. Slight, moderate, and severe vascular invasion were defined when one or two; three or four; and more than four vascular invasion sites, respectively, were seen in the submucosal layer of the stomach. Invasions were seen in the lower part of the resected stomach. Severe invasions were observed in several slices close to and far from the carcinoid tumors. It was thus difficult to determine from which of the three carcinoids the severe invasion had originated

Table 2. Classification of gastric carcinoid tumors

\begin{tabular}{|c|c|c|c|c|}
\hline & Type I & Type II & Type III & Our patient \\
\hline Tumor number & Multiple & Multiple & Solitary & Multiple \\
\hline Background & Type A gastritis & MEN-1, ZES & None & None \\
\hline Gastrin dependence & Yes & Yes & No & No \\
\hline Location & Fundus corpus & Fundus $\sim$ corpus & Fundus corpus & Body antrum \\
\hline Size & Small & Small & Large & Small \\
\hline Incidence (\%) [8] & 67.6 & 8.6 & 23.8 & $?$ \\
\hline Metastasis (\%) [8] & 7.6 & 12 & 65.4 & Positive LN metastasis \\
\hline
\end{tabular}

MEN-1, multiple endocrine neoplasia type 1; ZES, Zollinger-Ellison syndrome; LN, lymph node

of the upper body, and a 5-mm-diameter lesion in the lower body. Other multiple tiny (2- to 3-mm) nodules were also present. The histological findings of the gastric mucosa demonstrated moderate chronic gastritis without atrophic change. Helicobacter pylori was present. According to the histopathological findings of the resected specimen, the lesions exhibited multiple vascular invasions. In the lesser curvature of the upper and lower body, the original lesions seemed to arise from the submucosal layer and to grow invasively into the surrounding connective tissues. Immunohistochemistry, using CD31 and CD34 antibodies, showed lymphovascular invasion surrounding the tumors, and spreading widely in the mucosal or submucosal layers (Fig. 4). The largest carcinoid was the only one which had invaded the gastric mucosa, and we could not identify any other intramucosal lesion (Fig. 5). The histopathological diagnosis (Fig. 6) was a typical carcinoid tumor, the same as the findings of the preoperative biopsy specimen from the original lesions, without mitoses or prominent cellular atypia. p53 and c-erb B-2 protein expression were negative, and the $\mathrm{Ki}-67$ labeling index, which is often high in type- 3 gastric carcinoids, was less than $1 \%$. Tumor cells were not immunoreactive with gastrin, somatostatin, serotonin, or pancreatic polypeptide.

Endocrine cell hyperplasia, normally seen in multiple gastric carcinoid tumors, was not observed in the nontumorous gastric mucosa in this patient.

Of 45 lymph nodes dissected, metastasis was positive in 2 lymph nodes, of station no. 3 . 

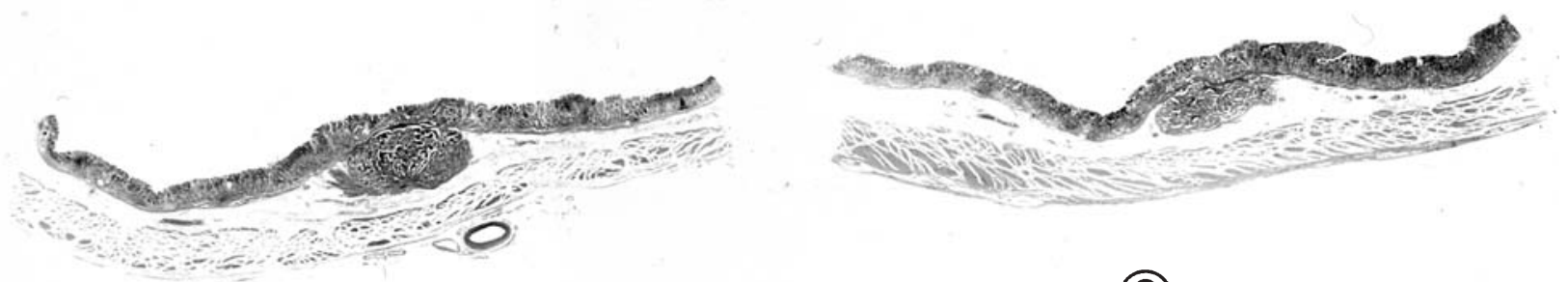

(1)
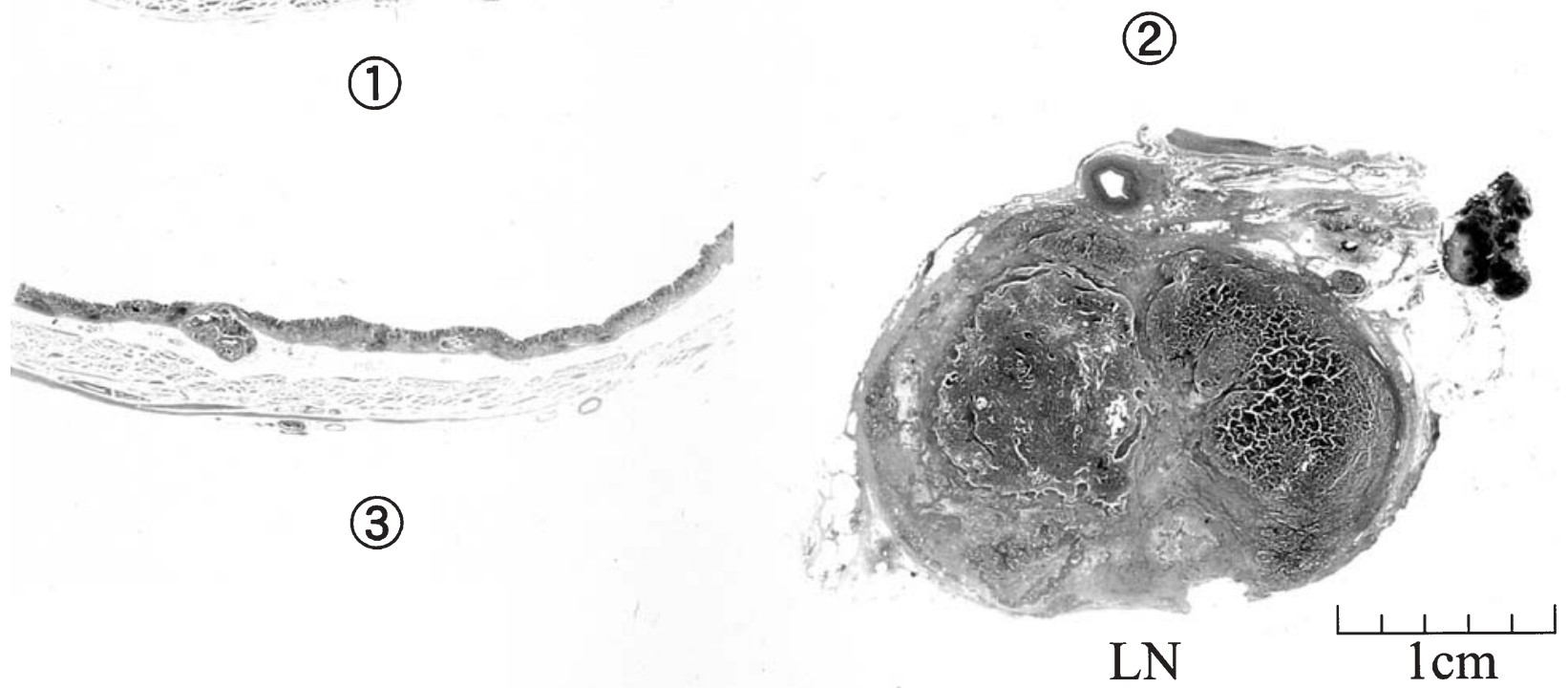

Fig. 5. Resected specimens viewed using low magnification. The carcinoid tumors were located mainly in the submucosal layer (1)-3). A large lymph node $(L N)$ was completely replaced by metastatic carcinoid tumor cells

\section{Discussion}

Gastric carcinoid tumors are classified into three types [2-7], as shown in Table 2. Gilligan et al. [10] recommended an endoscopic polypectomy following an antrectomy and also local excision for multiple carcinoid tumors in their decision tree for the management of gastric carcinoid tumors. Many reports recognize local excision or endoscopic resection for small gastric carcinoid tumors as a curative procedure [3-7]. Decreasing the serum gastrin level by performing an antrectomy is also known to diminish the number and size of types-I and -II multiple carcinoid tumors [11]. On the other hand, type-III carcinoid tumors should be treated in the same manner as gastric carcinoma, because of their aggressive behavior. Gastrectomy with systemic lymph node dissection is the recommended procedure for type-III carcinoid tumor.

Since 1993, we have performed laparoscopic wedge resection of the stomach for the diagnosis and treatment of submucosal tumors, such as myogenic tumors, Schwannomas, and gastrointestinal stromal tumors [12]. En-bloc excision of the tumor with intact surrounding tissue allows us to make a precise pathological examination and to judge accurately the need for any further treatment. Laparoscopic wedge resection is also indicated for solitary carcinoid tumors, but only when the tumor is smaller than several millimeters and has been preoperatively diagnosed as a type-I carcinoid tumor [13]. In patients in whom endoscopic mucosal resection is not indicated, we propose performing a distal gastrectomy, which can resect the tumors, lymph nodes, and antrum. In the present patient, with an extramural mass in the lesser curvature of the stomach that was first diagnosed as a submucosal tumor, a laparoscopic wedge resection was planned prior to the detection of the multiple carcinoid tumors. The intraoprerative findings, however, revealed multiple carcinoid tumors extending widely in the gastric wall, and, based on these findings, a total gastrectomy with $\mathrm{D} 1+\beta$ lymph node dissection was selected. This type of carcinoid tumor is rare and does not fit any of the three types of the recent classification. Not only the preoperative data but also the intraoperative findings should be considered carefully for the selection of the treatment procedure for carcinoid tumors. Three years have passed since the primary surgery in our patient, and no signs of recurrence have been identified in this 

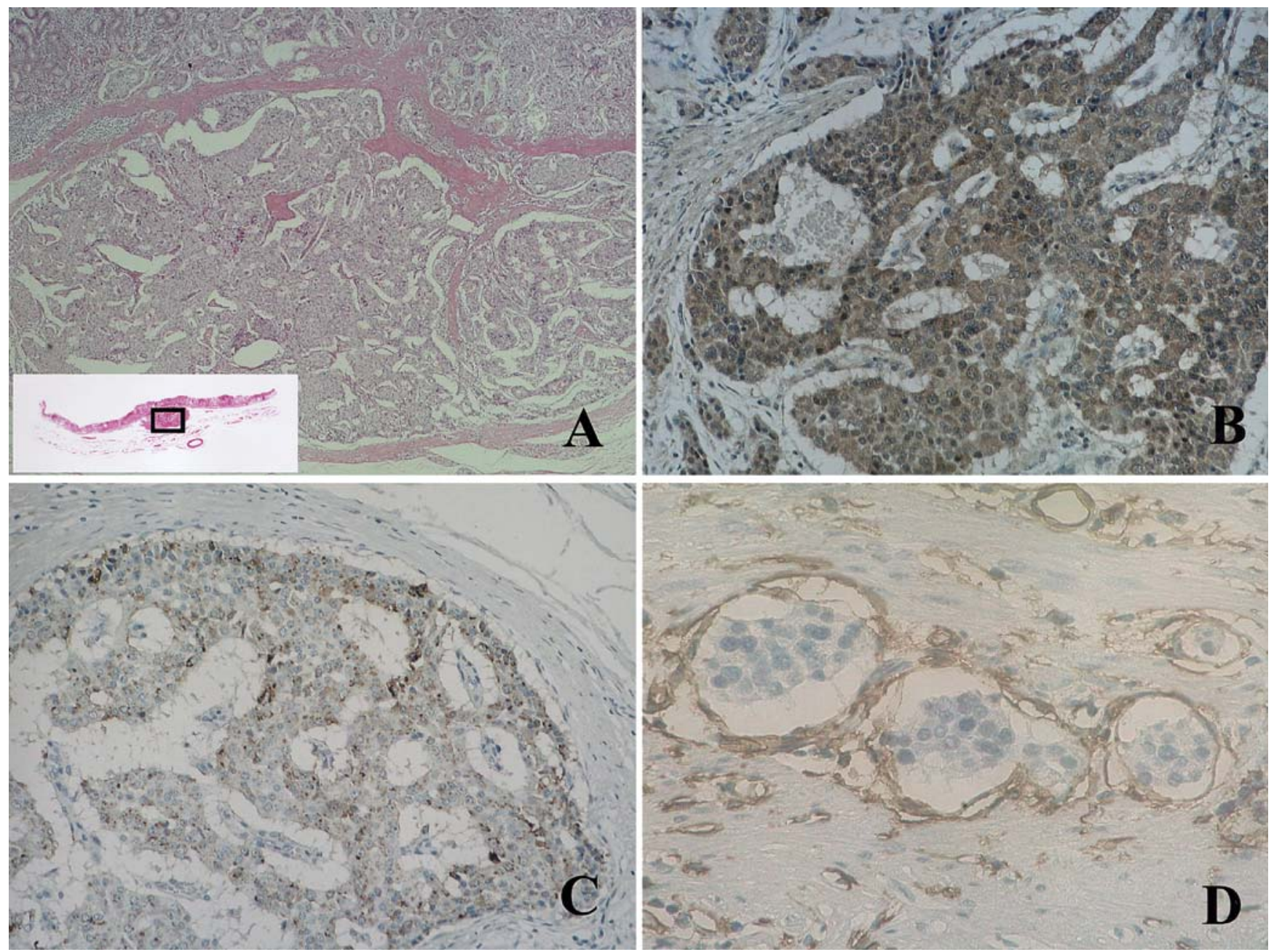

Fig. 6A-D. Pathological findings of the resected submucosal tumor (tumor no. (1) in Fig. 5). A H\&E staining; B neuronspecific enolase staining; $\mathbf{C}$ chromogranin A staining; and D CD34 staining. The neoplastic cells were arranged in nests or in a trabecular formation with well-vascularized stroma. $\mathrm{Nu}$ -

clei were round and regular. Mitotic figures were not seen (A). Tumor cells were immunoreactive with neuron-specific enolase (B) and chromogranin A (C). Massive vascular invasion was observed in the submucosal layer of the gastric wall between the tumors $(\mathbf{D})$

patient who, nevertheless, needs continued careful observation.

\section{References}

1. Bordi C, Costa A, Missale G. ECL cell proliferation and gastrin levels. Gastroenterology 1975;68:205-6.

2. Granberg D, Wilander E, Stridsberg M, Granerus G, Skogseid B, Oberg K. Clinical symptoms, hormone profiles, treatment, and prognosis in patients with gastric carcinoids. Gut 1998;43:223-8.

3. Bordi C, Yu JY, Baggi MT, Davoli C, Pilato FP, Baruzzi G, et al. Gastric carcinoids and their precursor lesions. A histologic and immunohistochemical study of 23 cases. Cancer 1991;67:1267-8.

4. Grigioni WF, Caletti GC, Gabrielli M, Marrano D, Villanacci V, Mancini A. Gastric carcinoids of ECL cells. Pathological and clinical analysis of eight cases. Acta Pathol Jpn 1985;35:361-75.

5. Lehy T, Cadiot G, Mignon M, Ruszniewski P, Bonfils S. Influence of multiple endocrine neoplasia type 1 on gastric endocrine cells in patients with Zollinger-Ellison syndrome. Gut 1992;331:2759.

6. Mueller J, Kirchner T, Mueller-Hermelink HK. Gastric endocrine cell hyperplasia and carcinoid tumors in atrophic gastritis type A. Am J Surg Pathol 1987;11:909-17.

7. Rindi G, Luinetti O, Cornagga M, Capella C, Solcia E. Three subtypes of gastric argyrophil carcinoid and the gastric neuroendocrine carcinoma: a clinicopathologic study. Gastroenterology 1993;104:994-1006.

8. Bordi C. Endocrine tumors of the stomach Pathol. Res Pract 1995;191:373-80.

9. Rindi G, Bordi C, Rappel S, La Rosa S, Stolte M, Solcia E. Gastric carcinoids and neuroendocrine carcinomas: pathogenesis, pathology, and behavior. World J Surg 1996;20:168-72.

10. Gilligan CJ, Lawton GP, Tang LH, West AB, Modlin IM. Gastric carcinoid tumors: the biology and therapy of an enigmatic and controversial lesion. Am J Gastroenterol 1995;90:338-52. 
11. Modlin IM, Gilligan CJ, Lawton GP, Tang LH, West AB, Darr U. Gastric carcinoid. Arch Surg 1995;130:250-6.

12. Otani Y, Ohgami M, Kubota T, Kumai K, Kitajima M, Mukai M. Laparoscopic wedge resection of gastric submucosal tumors. Surg Laparos, Endosc Percutaneous Techniques 2000;10:19-23.
13. Otani Y, Furukawa T, Suganuma K, Mukai M, Takami H, Kitajima M. Minimally invasive surgery for gastric carcinoid tumor. Biomed Pharmacother 2002;56:217s-21s. 